\title{
Periodontal Ligament Stem Cells-The Regeneration Front
}

\author{
Rajat Gothi ${ }^{1}$, Naveen Sangwan ${ }^{2}$, Ashutosh Kaushik ${ }^{3 *}$ and Neha Sikka ${ }^{4}$ \\ ${ }^{1}$ Senior lecturer, Department of Periodontology, Daswani Dental College and Research Centre, Ranpur, Kota, Rajasthan, India \\ ${ }^{2}$ Department of Periodontology, Consultant, Oral Health Department Oscar Hospital, Medical Modh, Rohtak, India \\ ${ }^{3}$ Senior lecturer, Department of Orthodontics, Daswani Dental College and Research Centre, Ranpur, Kota, Rajasthan, India \\ ${ }^{4}$ Senior Resident, PGIDS, Rohtak, Haryana, India
}

${ }^{*}$ Corresponding author: Dr. Ashutosh Kaushik, MDS, Department of Orthodontics and Dentofacial Orthopedics, Daswani Dental College, 781 A/ 28 , Bharat Colony, Rohtak-124001, Haryana, India, Tel: +919017176969; +919466460303; E-mail: dr.ashutoshkaushik@gmail.com

Rec Date: Dec 10, 2014; Acc Date: Dec 22, 2014; Pub Date: Jan 1, 2015

Copyright: (C) 2015 Gothi R et al., This is an open-access article distributed under the terms of the Creative Commons Attribution License, which permits unrestricted use, distribution, and reproduction in any medium, provided the original author and source are credited.

\begin{abstract}
The principal cells of the healthy, functional periodontal ligament are the differentiated cells and their progenitors. The differentiated cells are concerned with the synthesis and resorption of alveolar bone and the fibrous connective tissue of the ligament and cementum. It has been shown that small proportion of periodontal ligament cells are in mitosis at any given time. The cells of the periodontal ligament are also highly active metabolically; those of the rat molar turn the collagen over 5 times faster than do the fibroblast in the lamina propria of gingiva and 15 times faster than the fibroblast of skin. Progenitor cells exhibited marked differences in cytodifferentiation, suggesting that the progenitor cells that divide after wounding do not belong to a population of cells exhibiting uniform cytological characteristics, therefore it is unlikely that ultrastructural cytology can be used to identify these progenitor cells. Progenitor cells exhibited marked differences in cytodifferentiation, suggesting that the progenitor cells that divide after wounding do not belong to a population of cells exhibiting uniform cytological characteristics, therefore it is unlikely that ultrastructural cytology can be used to identify these progenitor cells. The evidence that these cells are present within the periodontal tissues was provided by McCulloch and coworkers by in vivo and histological studies.
\end{abstract}

Keywords: Stem cells; Periodontal ligament; Cementum

\section{Introduction}

The evidence that undifferentiated mesenchymal cells are present within the periodontal tissues was first provided by McCulloch and coworkers by in vivo and histological studies. An organism develops from a single cell going through various stages of development. However, only the early embryonic cells, recoverable from the fertilized oocyte and the descendents of the first two divisions are really totipotent i.e. capable of dividing without differentiating for a prolonged period in culture and able to form any kind of embryonic and extra-embryonic tissues [1] and not multipotent cells that can only form multiple lineages that constitute an entire tissue or tissues [2]. Regarding the field of Periodontics, the concept that stem cells may reside in the periodontal tissue was first proposed by Melcher in 1976 [3], who queried whether the three cell population of the periodontium (cementoblasts, alveolar bone cells and periodontal ligament fibroblast) were ultimately derived from a single population of ancestral cells or stem cells [4].

As the periodontium is an unusually complex tissue comprised of two hard (cementum and bone) and two soft (gingival and periodontal ligament) tissues, so once damaged, the periodontium has a limited capacity for regeneration [5]. Once, periodontitis becomes established, only therapeutic intervention has the potential to induce regeneration [6]. The complex series of events associated with periodontal regeneration involves recruitment of locally derived progenitor cells to the site which can subsequently differentiate into periodontal ligament-forming cells, mineral forming cementoblasts or bone forming osteoblasts [7].
Stem cells as defined by Austin Smith [2] is a cell that can continuously produce unaltered daughters and also has the ability to produce daughter cells that have different, more restricted properties. He also defined potency of stem cells as the range of commitment options available to a cell.

\section{A stem cell has two defining characteristics:}

The ability for indefinite self-renewal to give rise to more stem cells

The ability to differentiate into a number of specialized daughter cells to perform specific function [8].

So a stem cell can be of the following types:

\section{Classification of Stem Cells [2]}

Totipotent: Sufficient to form entire organism. Totipotency is seen in zygote and plant meristem cells; not demonstrated for any vertebrate stem cell.

Pluripotent: Able to form all the body's cell lineages, including germ cells, and some or even all extraembryonic cell types. Example: embryonic stem cells.

Multipotent: Can form multiple lineages that constitute an entire tissue or tissues. Example: haematopoietic stem cells.

Oligopotent: Able to form two or more lineages within a tissue. Example: a neural stem cell that can create a subset of neurons in the brain.

Unipotent: Forms a single lineage. Example: spermatogonial stem cells. 


\section{Classification of Dental Stem Cells [9]}

This classification is based on the differentiation potential of dental stem cells either into formation of dentin or periodontium-associated tissues.

$\mathrm{I}^{\text {st }}$ group associated with dental pulp consisting of:

Dental pulp stem cells (DPSC)

Stem cells of human exfoliated deciduous teeth (SHED)

Stem cells from apical papilla (SCAP)

$\mathrm{II}^{\text {nd }}$ group is associated with periodontium consisting of:

Periodontal ligament stem cells (PDLSC)

Dental follicle progenitor cells (DFPC)

\section{Periodontal Ligament Stem Cells (PDLSC)}

The principal cells of the healthy, functional periodontal ligament are the differentiated cells and their progenitors. The differentiated cells are concerned with the synthesis and resorption of alveolar bone and the fibrous connective tissue of the ligament and cementum. Consequently, the cells of the periodontal ligament may be divided into three main categories: synthetic cells, resorptive cells and progenitor cells.

Synthetic cells are formative cells like osteoblasts, fibroblasts, cementoblasts while resorptive cells are osteoclast, fibroblasts, cementoclasts and third category is the progenitor cells (generic term for any dividing cell with the capacity to differentiate including putative stem cells in which self-renewal has not yet been demonstrated). Progenitor cells for synthetic cells reside at least in part in the periodontal ligament, and the progenitor cells for resorptive cells (osteoclasts and cementoclast) originate from hematopoietic stem cells. In addition, to these other epithelial (Epithelial rest of malassez) and connective tissue cells, Masts cells and macrophages and other types of cells derived from hemopoietic line are present in the periodontal ligament [10]. It was only in the 1960s that scientists began to recognize the potential present in these stem cells when Mc Culloch and Till were able to demonstrate the presence of selfrenewing cells in mouse bone marrow. It was also during this decade that Joseph Altman and Gopal Das showed that adult neurogenesis was possible through stem cell activity in the brain [10]. High collagen turnover rate of periodontal ligament cells prompted researchers to carry out studies on periodontal ligament cells so as to ascertain whether these cells can be a prominent source of stem cells or not [10]. Melcher proposed the concept that stem cells may reside in the periodontal tissues [3]. It has been shown that small proportion of periodontal ligament cells are in mitosis at any given time. The cells of the periodontal ligament are also highly active metabolically; those of the rat molar turn the collagen over 5 times faster than do the fibroblast in the lamina propria of gingiva and 15 times faster than the fibroblast of skin [11].

Sodek studied collagen turnover in periodontal tissue by measurement of $\left[{ }^{3} \mathrm{H}\right]$ proline incorporated into newly synthesized and mature collagen in connective tissues, a highly efficient and rapid collagen turnover in rat periodontal tissues was demonstrated [12]. Gould et al. studied location of progenitor cells in periodontal ligament by creating wound in the alveolar process overlying the mesial root of the mandibular first molar expose periodontal ligament. The majority of the dividing cells were confirmed to be paravascular, and appeared to belong to two populations, one adjacent to bone, the other in the body of the ligament. A third population, not paravascular, lay adjacent to cementum [13]. In the year 1980, Gould et al studied the migration and division of progenitor cell populations in periodontal ligament after wounding the periodontal ligament of the lower first molar in mice using the techniques of radioautography and grain counting [14]. They showed that: Mitotic cells (presumably progenitor cells) are on an average located significantly closer to blood vessels than are interphase cells. Progenitor cells exhibited marked differences in cytodifferentiation, suggesting that the progenitor cells that divide after wounding do not belong to a population of cells exhibiting uniform cytological characteristics, therefore it is unlikely that ultrastructural cytology can be used to identify these progenitor cells [1]. The evidence that these cells are present within the periodontal tissues was provided by Mc Culloch and coworkers by in vivo and histological studies [11]. Mc Culloch et al. conducted a study to examine whether the endosteal spaces of alveolar bone communicate with the periodontal ligament and do they contribute to its cell populations. The data was consistent with the hypothesis that cells migrated from endosteal spaces into the periodontal ligament and there express the phenotype for osteoblasts or cementoblasts [11]. Somerman et al. in 1988 compared human periodontal ligament cells and gingival fibroblasts, both derived from the same patient, same passage, in-vitro. The results from this study indicate that periodontal ligament cells exhibit characteristics significantly distinct from those of gingival fibroblasts in-vitro, i.e., greater protein and collagen production and higher alkaline phosphatase activity [15]. Giannopoulou et al. studied gingival and periodontal ligament fibroblasts derived from five healthy subjects were isolated and compared in-vitro. Epithelial cells increased the proliferation of both GF and PDLF but had no effect on their biosynthetic activity. These in-vitro results may better explain the in vivo functional differences between GF and PDLF [16]. In the event of injury to the periodontium these mesenchymal stem cells could be activated towards terminal differentiation and tissue repair or regeneration. In order to identify putative periodontal ligament stem cells (PDLSC), various techniques which have been used to characterize bone marrow stromal stem cells (BMSSC) and dental pulp stem cells (DPSC) were employed, including cell culture, magnetic and fluorescence activated cell sorting, immunohistochemistry, RT-PCR, western and northern blot analyses. When plated under the same growth conditions as for BMSSCs and DPSCs, PDL derived cells were found to generate clonogenic adherent cell colonies, where the incidence of fibroblastic colony forming unit (CFUF), was greater than that reported for BMSSCs and DPSCs. Therefore, these cells were termed periodontal ligament stem cells, PDLSCs by Seo et al. [17]. Seo et al. in 2005 utilized human periodontal ligament to test the hypothesis that cryopreserved human periodontal ligament contains retrievable postnatal stem cells. The study demonstrated that human post-natal stem cells can be recovered from cryopreserved human periodontal ligament, thereby providing a practical clinical approach for the utilization of frozen tissues for stem cell isolation [18]. Shi et al. studied identification, characterization, and potential application of mesenchymal stem cells (MSC) derived from human dental tissues. Their study showed that MSC were identified in adult human dental pulp (dental pulp stem cells, DPSC), human primary teeth (stem cells from human exfoliated deciduous teeth, SHED), and periodontal ligament (periodontal ligament stem cells, PDLSC) by their capacity to generate clonogenic cell clusters in culture. PDLSC were also found to express the tendon specific marker, Scleraxis [19]. Tomokiyo et al. [20] aimed to establish a human PDLcommitted stem cell line and investigate the effects of basic fibroblast 
growth factor (bFGF) on the osteoblastic differentiation of the cells. These results suggest that cell line $1-17$ is a PDL-committed stem cell line and that bFGF exerts dualistic (i.e., promoting and inhibitory) effects on the osteoblastic differentiation of cell line $1-17$ based on its differentiation stag [20]. Feng et al. in this study, retrospectively examined feasibility and safety of reconstructing the periodontal intrabony defects with autologous periodontal ligament progenitor (PDLP) implantation in three patients. Study demonstrated clinical and experimental evidences supporting a potential efficacy and safety of utilizing autologous PDL cells in the treatment of human periodontitis [21]. Iwata et al. aim was to know whether periodontal ligament is a reliable source for periodontal regeneration and so they followed an optimal protocol for the extraction, expansion and characterization of human PDL (hPDL) cells was examined for clinical trials. They found that hPDL cells frequently differentiated into cementoblastic/ osteoblastic lineage [22]. Park et al. had an objective to study mesenchymal stem cells (MSC) that could be isolated from healthy periodontal ligaments (PDL). Their aim was to isolate and characterize human PDL stem cells (hPDLSCs) from inflamed PDL tissue, and to evaluate their regenerative potential. ihPDLSCs were successfully isolated and characterized as MSCs. Both ihPDLSCs (inflamed) and hPDLSCs were successfully differentiated under osteogenic/cementogenic and adipogenic microenvironment. The proliferative potential did not differ between healthy hPDLSCs and ihPDLSCs and concluded that ihPDLSCs could be successfully isolated from inflamed PDL tissue, and they retained the regenerative potential for cementum and related periodontal ligament tissue [23]. Choi et al. [24] used immunophenotyping and fluorescence-activated cell sorting analysis and found that certain mesenchymal stem cell markers (CD44, CD73, CD90, CD146 and CD166) were heavily expressed in human adult dental pulp cells (hDPCs), periodontal ligament stem cells (hPDLSCs) and gingival fibroblasts (hGFs). However, on treating with osteogenic additives, mineralization was only observed in hDPCs and hPDLSCs, but not in the hGFs. The expression of dentinogenic markers (dentin sialophosphoprotein and dentin matrix protein-1), decreased during extended culture of the hDPCs and hPDLSCs. They concluded that the mere presence of mesenchymal stem cell surface proteins in hGFs does not mandate the differentiation capability during culture. Also, the expression of dentinogenic markers by hDPCs was not affected during co-culture with hPDLSCs and/or hGFs but it was diminished during extended culture.

\section{Discussion}

Application of tissue-engineering to periodontal regeneration will need to utilize the regenerative capacity of cells residing within the periodontium and would involve the isolation of such cells and their subsequent proliferation within a three-dimensional framework with implantation into the defect. Recent advances in growth factor biology and biodegradable polymer constructs have set the stage for successful tissue engineering of cartilage, bone and related tissues of which the periodontium could be considered a prime candidate for such procedures [25].

An appropriate cell source is another important requirement for tissue engineering. Exogenous cells are required when the proliferation of the precursor cells is impeded, or they have been greatly diminished by previous surgery or concomitant disease. Therefore the required cells must be identified, isolated and produced in sufficient numbers. So, here comes the role of stem cells which is defined as a cell that can continuously produce unaltered daughters and also has the ability to produce daughter cells that have different, more restricted properties [2]. The ideal stem cell for stem cell-based therapy still remains to be elucidated. In the past, although various types of stem cells have been isolated from teeth (dental pulp stem cells (P-MSCs), periodontal ligament stem cells (PDL-MSCs), stem cells from exfoliated human deciduous teeth (SHED), stem cells from root apical papilla (SCAP), dental follicle progenitor stem cells, Gingival Mesenchymal stem cells (GMSCs) [26-29], orofacial bone/bone-marrow-derived MSCs [26-29], Tooth germ stem cells (TGSCs) [26-29], MSCs from periapical lesions (PL-MSCs) [26-29], Oral mucosal lamina propriaderived progenitor cells (OMLP-PCs) Ding et al. [30] and granulation tissue from tooth extraction socket [31] but the ideal stem cell is still questionable, including, which may be a good stem cell type for stem cell-based therapy in certain disease, especially when they origin from neural crest is considered [32]. Hakki et al. [32] and Lei et al. [33] conducted studies to compare P-MSCs and PDL-MSCs and found higher proliferation potential and telomerase activity in P-MSCs. They concluded that the different type of dental stem cells have different cellular behavior, thus, should be carefully chosen based on location and purpose.

Periodontal ligament stem cells (PDL-MSC): Further class of dental ectomesenchymal stem cells is PDL stem cells, which were isolated from the root surface of extracted teeth. These cells could be isolated as plastic-adherent, colony-forming cells, but display a low potential for osteogenic differentiation under in-vitro conditions. PDL stem cells differentiate into cells or tissues very similar to the periodontium [17].

Various procedures have been attempted to achieve periodontal regeneration that is restoration of lost supporting tissues including new alveolar bone, new cementum, and new periodontal ligament. Despite conclusive evidence that some regeneration may occur following regenerative procedures, complete regeneration has been an elusive goal.

The multilineage differentiation potential of PDLSC including adipogenic, chondrogenic, osteogenic potential has been demonstrated in various studies conducted by Seo et al. [18]; Gronthos et al. [34]; Gay et al. [35].

Gronthos et al. [34] demonstrated that CD106+ ovine PDLSCs demonstrated the capacity to form adherent clonogenic clusters of fibroblast-like cells when plated at low densities in-vitro. EX-vivo expanded ovine PDLSCs exhibited a high proliferation rate in-vitro and expressed a phenotype (CD44+, CD166+, CBFA-1+, collagen-I+, bone sialoprotein+) consistent with human-derived PDLSCs. Furthermore, cultured ovine PDLSCs expressed high transcript levels of the ligament/tendon-specific early transcription factor scleraxis. The results from this study suggest that ovine PDLSCs may potentially be used as a novel cellular therapy to facilitate successful and more predictable regeneration of periodontal tissue using an ovine preclinical model of periodontal disease as a prelude to human clinical studies [34].

However, the extent to which in-vitro differentiation capacity is relevant remains unclear and at present, remains the key issue for the field. More recently neurogenic differentiation from murine demonstrating neural crest markers have been demonstrated by Techawattanawisal et al. [35] in 2007 who showed that isolated multipotent stem cells from rat periodontal ligament (PDL) using neurosphere-forming culture system and enzymatically dissociated PDL cells were cultured in serum-free basal medium containing EGF, bFGF, and LIF. Free-floating spheres expressing nestin, GFAP, and 
vimentin were formed by 7 days of the culture and data gathered indicated that PDL-derived spheres contained multipotent adult stem cells capable of differentiating into both neural and mesodermal progeny. This was the first report of the isolation of PDL-derived stem cells with primitive neural crest stem cell features [36].

While differentiation from human PDLSC demonstrating neural crest markers have been demonstrated by Huang et al. in 2010 [37] who showed that subpopulations of PDL cells expressed embryonic stem cell markers (Oct4, Sox2, Nanog and Klf4) and a subset of neural crest markers (Nestin, Slug, p75 and Sox10). Such PDL cell subpopulations exhibited the potential to differentiate into neurogenic, cardiomyogenic, chondrogenic and osteogenic lineages. Furthermore, preliminary evidence suggesting insulin production of PDL cells might be indicative of the generation of cells of the endodermal lineage [36].

PDLSC are shown to have osteogenic potential similar to BMSC as suggested by in-vitro studies demonstrating mineralized nodule formation under appropriate culture conditions, although in-vitro mineralization cannot be considered a definitive indicator of in vivo osteogenic differentiation potential in true sense. Many studies have reported that PDLSC isolates to have lower osteogenic potential than BMSC and also dental pulp derived cell.

Gay et al. [35] isolated and characterized PDLSC to assess their capability to differentiate into bone, cartilage and adipose tissue and found that human PDL tissue contains about 27\% STRO-1 positive cells with $3 \%$ strongly positive. In osteogenic cultures ALP (alkaline phosphatise) was observed by day-7 in BMSC and day-14 in PDLSC. BSP expression was detectable by day-7; with more intense staining in PDLSC cultures. In adipogenic cultures both cell populations showed positive Oil Red $\mathrm{O}$ staining by day-25 with PPAR 2 and LPL expression. By day-21, both BMSC and PDLSC chondrogenic induced cultures expressed collagen type II and glycosaminoglycans. They concluded that the PDL contains SC that have the potential to differentiate into osteoblasts, chondrocytes and adipocytes, comparable with previously characterized BMSC. This adult PDLSC population can be utilized for potential therapeutic procedures related to PDL regeneration [36]. Li et al. [38] proved that the periodontal stem cells and the autologous Schwann cells exhibited similar therapeutic effects in case of transplantation after mental nerve injury.

Trubiani et al. study indicated that periodontal ligament can be an easily and efficient autologous source of stem cells with a high expansion capacity and ability to differentiate in osteogenic cells that can colonize and grow connected to bio-compatible scaffold. It can be suggested that the use of PDL-MSCs for generating graft biomaterials is advantageous for bone tissue engineering in regenerative dentistry [39].

Seo et al. showed inability of PDLSC to form bone in vivo17 as opposed to reports by others such as Kim et al. [40] who evaluated the potential of BMSCs and PDLSCs on alveolar bone regeneration in a canine peri-implant defect model. The results indicated that transplantation of autologous canine BMSCs and PDLSCs with HA/TCP carriers is effective for bone regeneration in surgically created periimplant saddle-like defects compared to cell-free HA/TCP. Furthermore, the transplanted BMSCs and PDLSCs remained at the grafted area and presumably participated in bone regeneration [40].

PDL tissues are clinically accessible in routine clinical practices, such as tooth extraction, possibly providing a readily available source of stem cells for clinical periodontal regenerative therapy. However, little is known about the characteristics of PDL progenitor cells/stem cells because PDL is composed of heterogeneous cell populations, and thus far, no highly purified PDLSC clone has been established from human PDL tissue. Consequently, even if the stemness (self-renewal and potency) of cultured PDLSCs and expression of SC markers were confirmed, the canine PDLSCs used in the present study are of heterogenous origin, and this limitation may have had an effect on the regeneration results.

More recently, the molecular marker periodontal ligament associated protein-1 (PLAP-1)/asporin has been identified by Yamada et al. [41] as being specific to periodontal ligament phenotype and to inhibit mineralization. The transcription of PLAP-1 was upregulated along with the cytodifferentiation process of PDL cells and downregulated when the process was arrested by FGF-2. This suggests that PLAP-1 expression is closely associated with the process of cytodifferentiation of PDL cells [42].

Let us consider the immunomodulatory properties of PDLSC as there is much in-vitro evidence indicating that various MSC are hypoimmunogenic, and also modulate the $\mathrm{T}$ cell response, independent of major histocompatibility complex (MHC) expression. In-vitro, human MSCs express intermediate levels of human leukocyte antigen (HLA), MHC class I molecules, are negative for MHC class II molecules (but can be induced to express MHC class II by interferon gamma), and lack co- stimulatory molecules B7-1, B7-2, CD4O, and $\mathrm{CD} 4 \mathrm{O}$ ligand. Consequently, these cells may escape recognition by alloreactive T cells. Ding et al. [30] in their study, examined the immunomodulatory properties of PDLSCs as candidates for new allogeneic stem cell-based therapies. Human PDLSCs displayed cell surface marker characteristics and differentiation potential similar to bone marrow stromal stem cells (BMSSCs) and dental pulp stem cells (DPSCs). PDLSCs, BMSSCs, and DPSCs inhibited peripheral blood mononuclear cell (PBMNC) proliferation stimulated with mitogen or in an allogeneic mixed lymphocyte reaction (MLR). These results suggest that PDLSCs, BMSSCs, DPSCs, and GFs possess immunosuppressive properties mediated, in part, by soluble factors, produced by activated PBMNCs [43].

However, very significantly, contradictory in vivo findings have been noted, showing a strong cellular immune response to transplanted MSC, indicating a possible alteration of antigen expression in vivo. Hence, the in vivo utility of allogeneic PDLSC remains highly questionable, in line with earlier reports showing that human allogeneic tooth transplantations cause rejection due to immunemediated osteoclast activation. Although a recent animal study showed evidence that, after allogeneic tooth transplantation, the donor periodontal tissue was replaced and regenerated by host cells without exhibiting a MHC -mediated host immune response, findings from invitro and inbred animal studies need to considered with caution and validated in appropriate preclinical trials. Further studies are needed to investigate any potential value of PDLSC supported immunomodulatory activity in actual clinical settings [43].

Tissue engineering, aimed at developing techniques for the fabrication of new tissues to replace damaged or diseased tissues, is based on principles of cell biology, developmental biology and biomaterials. Recent advances in growth factor biology and biodegradable polymers have set the stage for successful tissue engineering of cartilage, bone and other tissues, of which the periodontium could be considered a prime candidate for such procedures. Studies to date have shown that periodontal ligament cells can he transplanted into periodontal defects with no adverse 
Page 5 of 7

immunologic or inflammatory consequences [44-46]. More recently, cementoblasts as well as various periodontal cells transfected with vectors for over expression of various growth factors have been investigated in periodontal tissue engineering models [47]. A tissue engineering strategy for periodontal regeneration that exploits the regenerative capacity of stem cells residing within the periodontium is an attractive prospect. By using such an approach the need for recruitment of cells to the site is negated and the predictability of the outcome may be enhanced.

PDLSC represent a novel stem cell population, in terms of in vivo capacity to develop into cementoblast-like cells, cementum and periodontal ligament- like tissue, as evidenced positively in preclinical studies [16] using a rodent model, demonstrating a cementum/PDLlike complex generated in surgically created periodontal defects by transplanting in-vitro expanded human PDLSCs in a ceramic particle scaffold [17]. There is a similar report of histologic periodontal regeneration in vivo by expanded autologous PDLSC in a swine model [20]. Another porcine model study reports transplanting autologous swine PDLSCs, which lead to the generation of a root/periodontal complex capable of supporting a porcelain crown, resulting in normal tooth function. Vecchiatini et al. [47] studied the efficiency of culture of mesenchymal stem cells obtained from periodontal ligament, coated with alginate microbeads in bioreactor system and found it as a promising technique to be used in different dental applications. Besides periodontal regeneration, another potential application of PDLSCs is in the area of hybrid 'tooth engineering' in combination with other stem and progenitor cell populations and scaffolds.

Periodontal tissue engineering using PDLSC conventionally needs 3 -D biomaterial scaffold technology $[48,49]$ that can closely mimic the effect of extracellular matrix (ECM) derived signals for optimal differentiation, however, there are inherent shortages in current scaffold technology. This has led to the development scaffold-free methodology for PDLSC transplantation, such as cell sheets and recently, a promising novel 3D human PDLSC cell pellet, which selfsecretes ECM and has favorable fabrication and handling, demonstrated the formation of a cementum, PDL-like complex on transplantation into immunocompromised mice [50].

It is of interest to note that cryopreserved PDLSC may be collected and saved for future use through preservation techniques such as freezing in liquid nitrogen. Seo et al. reported that periodontal ligament, preserved frozen in liquid nitrogen, generated high proliferative PDLSC, although the number of PDLSC colonies derived was decreased in comparison with freshly isolated tissue samples. Thus, in future, use of cryopreserved PDLSCs could widen the application arena. Transplantation of these cells, which can be obtained from an easily accessible tissue resource and expanded ex vivo, might hold promise as a therapeutic approach for reconstruction of tissues destroyed by periodontal diseases [17].

It has also been reported that high proliferating periodontal ligament stem cells are representative of only a minor proportion of the cells which can be expanded in-vitro over successive cell passages. Also these cells undergo senescence and hence have a finite lifespan. This appears to be a feature of most postnatal stem cells that markedly separates them from embryonic stem cells, which are virtually immortal [14].

The immortal nature of embryonic stem cells has been related to their high expression of the enzyme telomerase, which is found to be absent in many mesenchymal stem cells. This may therefore be an important factor for prolonging cellular senescence, leading to increased proliferation and survival rate. It has been demonstrated that if bone marrow stromal stem cells are induced to express active telomerase their lifespan was increased almost threefold [43].

Thus, the potential exists to develop strategies to genetically manipulate ex vivo expanded mesenchymal stem cells, such as periodontal ligament stem cells, to enhance and regulate their growth properties with a view to clinical applications. In 2005, Fujita et al. demonstrated immortilisation of human periodontal ligament cells by transduction with the hTERT gene [51]. The recent protocol advocated by Elcin YM et al. suggests that the guided differentiation of pluripotent human embryonal stem cells (hESCs) by coculture with adult PDLFs, results in conversion to fibroblastic and osteoblastic lineages [52]. Choi et al. [50] suggested that several genes related to proliferation or migration were expressed when hPDL cells (human periodontal ligament stem cell) were cultured in non-osteogenic medium. Also, expression of the genes related to calcium/iron/metal ion binding or homeostasis and cell viability were increased at the stage of matrix maturation, and expression of the genes related to apoptosis, angiogenesis, and adipogenesis were increased at the early stage of mineralization or the late stage of matrix maturation when hPDL cells were cultured in osteogenic medium [53,54]. They suggested that when appropriately triggered, the stem cells in the hPDL differentiate into osteoblasts/ cementoblasts, and, the genes related to calcium binding such as PDE1A, PCDH9, which was strongly expressed at the stage of matrix maturation, may be associated with differentiation of the hPDL cells into osteoblasts/ cementoblasts.

Once the periodontal ligament stem cells have been identified and there growth and differentiation potential recognized, it is seems logical that autologous periodontal ligament stem cells can be cultured within a suitable delivery scaffold, in conjunction with the growth and differentiation factors present in an autologous blood clot, will lead to new periodontal tissue attachment via a tissue engineering approach [5]. The concept of cell seeding i.e. incorporation of cells in implantable matrices is currently being used in periodontics. It has been shown that periodontal ligament cells can be transplanted into periodontal defects with no adverse immunologic or inflammatory consequences $[44,52]$. Therefore tissue engineering strategy for periodontal regeneration that exploits the regenerative capacity of stem cells residing within the periodontium is an attractive proposition. By using such an approach the need for recruitment of cells to the site is negated and the predictability of the outcome may be enhanced. Xu et al. [55] found that 1\% PRP significantly increased the osteogenic differentiation of PDLSCs by stimulating the production of extracellular matrix proteins and the expression of the osteogenic genes ALP, Runx2, Col-1 and OCN.

\section{Conclusion}

A significant research is ongoing at an unprecedent rate on dental stem cells as these appear to be a promising source for treatment of various diseases and aboon in the regenerative treatment. The dental stem cells can be conveniently obtained following minimally or nonivasive procedures. As the dental stem cells are adult stem cells, thus, there are feeble chances of transplant rejection. However, these cells are heterogenous. So, in order to specifically identify and isolate the subset of stem cells within the periodontium, marker identification is essential. Besides, as adult stem cells have the property of plasticity like other cell strains such as BMSSCS, SHEDS, so precursor of cells from 
human dental follicle of wisdom teeth might be in the future, genetically modified in-vitro so that they will be able to differ in periodontal tissue cells before they are transplanted in vivo. Also, the understanding of the regulation of genetic expression by PDL-MSCs is must. Control of these signals in correct pattern is desirable to produce the required tissue in-vitro as well as in-vivo. The long term fate of the transplanted cells is still questionable.

Although new studies will be necessary to put in practice these alternatives therapies, at this moment the better understanding of stem cells and their possible role in tissue regeneration can help develop new approaches for a more predictable management of periodontal defects.

\section{References}

1. Rimondini L, Mele S (2009) Stem cell technologies for tissue regeneration in dentistry. Minerva Stomatol 58: 483-500.

2. Smith A (2006) A glossary for stem-cell biology. Nature 441: 1060.

3. Melcher AH (1985) Cells of periodontium: their role in the healing of wounds. Ann R Coll Surg Engl 67: 130-131.

4. Silvério KG, Benatti BB, Casati MZ, Sallum EA, Nociti FH Jr (2008) Stem cells: potential therapeutics for periodontal regeneration. Stem Cell Rev 4: 13-19.

5. Bartold PM, Shi S, Gronthos S (2006) Stem cells and periodontal regeneration. Periodontol 2000 40: 164-172.

6. Bartold PM, McCulloch CA, Narayanan AS, Pitaru S (2000) Tissue engineering: a new paradigm for periodontal regeneration based on molecular and cell biology. Periodontol 24: 253-269.

7. Bartold PM, Narayanan AS (1998). Periodontal regeneration. In: biology of periodontal connective tissue, chapter 11; Quintessence publishing, Chicago.

8. Department of Health and Human Services (2001) Stem Cells: Scientific Progress and Future Research Directions.

9. Morsczeck C, Schmalz G, Reichert TE, Völlner F, Galler K, et al. (2008) Somatic stem cells for regenerative dentistry. Clin Oral Investig 12: 113-118.

10. Bhaskar SN (2004) Orban's Oral histology and embryology. (11edtn) Elsevier, St. Louis, Missouri.

11. McCulloch CA, Nemeth E, Lowenberg B, Melcher AH (1987) Paravascular cells in endosteal spaces of alveolar bone contribute to periodontal ligament cell populations. Anat Rec 219: 233-242.

12. Sodek J (1976) A new approach to assessing collagen turnover by using a micro-assay. A highly efficient and rapid turnover of collagen in rat periodontal tissues. Biochem J 160: 243-246.

13. Gould TR, Melcher AH, Brunette DM (1977) Location of progenitor cells in periodontal ligament of mouse molar stimulated by wounding. Anat Rec 188: 133-141.

14. Gould TR, Melcher AH, Brunette DM (1980) Migration and division of progenitor cell populations in periodontal ligament after wounding. J Periodontal Res 15: 20-42.

15. Somerman MJ, Archer SY, Imm GR, Foster RA (1988) A comparative study of human periodontal ligament cells and gingival fibroblasts in vitro. J Dent Res 67: 66-70.

16. Giannopoulou C, Cimasoni G (1996) Functional characteristics of gingival and periodontal ligament fibroblasts. J Dent Res 75: 895-902.

17. Seo BM, Miura M, Gronthos S, Bartold PM, Batouli S, et al. (2004) Investigation of multipotent postnatal stem cells from human periodontal ligament. Lancet 364: 149-155.

18. Seo BM, Miura M, Sonoyama W, Coppe C, Stanyon R, et al. (2005) Recovery of stem cells from cryopreserved periodontal ligament. J Dent Res 84: 907-912.
19. Shi S, Bartold PM, Miura M, Seo BM, Robey PG, et al. (2005) The efficacy of mesenchymal stem cells to regenerate and repair dental structures. Orthod Craniofac Res 8: 191-199.

20. Tomokiyo A, Maeda H, Fujii S, Wada N, Shima K, et al. (2008) Development of a multipotent clonal human periodontal ligament cell line. Differentiation 76: 337-347.

21. Feng F, Akiyama K, Liu Y, Yamaza T, Wang TM, et al. (2010) Utility of PDL progenitors for in vivo tissue regeneration: a report of 3 cases. Oral Dis 16: 20-28.

22. Iwata T, Yamato M, Zhang Z, Mukobata S, Washio K, et al. (2010) Validation of human periodontal ligament-derived cells as a reliable source for cytotherapeutic use. J Clin Periodontol 37: 1088-1099.

23. Park JC, Kim JM, Jung IH, Kim JC, Choi SH, et al. (2011) Isolation and characterization of human periodontal ligament (PDL) stem cells (PDLSCs) from the inflamed PDL tissue: in vitro and in vivo evaluations. J Clin Periodontol 38: 721-731.

24. Choi JK, Hwang HI, Jang YJ (2015) The efficiency of the in vitro osteo/ dentinogenic differentiation of human dental pulp cells, periodontal ligament cells and gingival fibroblasts. Int J Mol Med 35: 161-168.

25. Bright R, Hynes K, Gronthos S, Bartold PM (2014) Periodontal ligamentderived cells for periodontal regeneration in animal models: a systematic review. J Periodontal Res.

26. Tan J, Xu X, Lin J, Fan L, Zheng Y, et al. (2014) Dental Stem Cell in Tooth Development and Advances of Adult Dental Stem Cell in Regenerative Therapies. Curr Stem Cell Res Ther.

27. Bojic S, Volarevic V, Ljujic B, Stojkovic M (2014) Dental stem cells-characteristics and potential. Histol Histopathol 29: 699-706.

28. Hynes K, Menicanin D, Gronthos S, Bartold PM (2012) Clinical utility of stem cells for periodontal regeneration. Periodontol 2000 59: 203-227.

29. Liu J, Yu F, Sun Y, Jiang B, Zhang W, et al. (2014) Characteristics and Potential Applications of Human Dental Tissue-Derived Mesenchymal Stem Cells. Stem Cells.

30. Ding G, Niu J, Wei F (2014) Current understanding of orofacial tissue derived mesenchymal stem cells: an immunological perspective. Histol Histopathol.

31. Nakajima R, Ono M, Hara ES, Oida Y, Shinkawa S, et al. (2014) Mesenchymal stem/progenitor cell isolation from tooth extraction sockets. J Dent Res 93: 1133-1140.

32. Hakki SS, Kayis SA, Hakki EE, Bozkurt SB, Duruksu G, et al. (2014) Comparison of MSCs Isolated From Pulp and Periodontal Ligament. J Periodontol.

33. Lei M, Li K, Li B, Gao LN, Chen FM, et al. (2014) Mesenchymal stem cell characteristics of dental pulp and periodontal ligament stem cells after in vivo transplantation. Biomaterials 35: 6332-6343.

34. Gronthos S, Mrozik K, Shi S, Bartold PM (2006) Ovine periodontal ligament stem cells: isolation, characterization, and differentiation potential. Calcif Tissue Int 79: 310-317.

35. Techawattanawisal W, Nakahama K, Komaki M, Abe M, Takagi Y, et al. (2007) Isolation of multipotent stem cells from adult rat periodontal ligament by neurosphere-forming culture system. Biochem Biophys Res Commun 357: 917-923.

36. Huang CY, Pelaez D, Dominguez-Bendala J, Garcia-Godoy F, Cheung HS (2009) Plasticity of stem cells derived from adult periodontal ligament. Regen Med 4: 809-821.

37. Gay IC, Chen S, MacDougall M (2007) Isolation and characterization of multipotent human periodontal ligament stem cells. Orthod Craniofac Res 10: 149-160.

38. Li B, Jung HJ, Kim SM, Kim MJ, Jahng JW, et al. (2013) Human periodontal ligament stem cells repair mental nerve injury.Neural Regen Res 8: 2827-2837.

39. Trubiani O, Orsini G, Zini N, Di Iorio D, Piccirilli M, et al (2008). Regenerative potential of human periodontal ligament derived stem cells on three-dimensional biomaterials: A morphological report. J Biomed Mater Res A 87: 986-993. 
Citation: Gothi R, Sangwan N, Kaushik A, Sikka N (2015) Periodontal Ligament Stem Cells-The Regeneration Front. Dentistry 5: 275. doi:

40. Kim SH, Kim KH, Seo BM, Koo KT, Kim TI, et al. (2009) Alveolar bone regeneration by transplantation of periodontal ligament stem cells and bone marrow stem cells in a canine peri-implant defect model: a pilot study. J Periodontol 80: 1815-1823.

41. Yamada S, Ozawa Y, Tomoeda M, Matoba R, Matsubara K, et al. (2006) Regulation of PLAP-1 expression in periodontal ligament cells. J Dent Res 85: 447-451.

42. Acharya A, Shetty S, Deshmukh V (2010). Periodontal ligament stem cell: An overview. J Oral Biosci 52: 272-282.

43. Van Dijk LJ, Schakenraad JM, van der Voort HM, Herkströter FM Busscher HJ (1991) Cell-seeding of periodontal ligament fibroblasts. A novel technique to create new attachment. A pilot study. J Clin Periodontol 18: 196-199.

44. Lang H, Schüler N, Nolden R (1998) Attachment formation following replantation of cultured cells into periodontal defects--a study in minipigs. J Dent Res 77: 393-405.

45. Malekzadeh R, Hollinger JO, Buck D, Adams DF, McAllister BS (1998) Isolation of human osteoblast-like cells and in vitro amplification for tissue engineering. J Periodontol 69: 1256-1262.

46. Zhao M, Jin Q, Berry JE, Nociti FH Jr, Giannobile WV, et al. (2004) Cementoblast delivery for periodontal tissue engineering. J Periodontol 75: 154-161.

47. Vecchiatini R, Penolazzi L, Lambertini E, Angelozzi M, Morganti C, et al. (2014) Effect of dynamic three-dimensional culture on osteogenic potential of human periodontal ligament-derived mesenchymal stem cells entrapped in alginate microbeads. J Periodontal Res 2014.
48. Kim JH, Park CH, Perez RA, Lee HY, Jang JH, et al. (2014) Advanced Biomatrix Designs for Regenerative Therapy of Periodontal Tissues. J Dent Res 93: 1203-1211.

49. Ivanovski S, Vaquette C, Gronthos S, Hutmacher DW, Bartold PM (2014) Multiphasic scaffolds for periodontal tissue engineering. J Dent Res 93: 1212-1221.

50. Choi HD, Noh WC, Park JW, Lee JM, Suh JY (2011) Analysis of gene expression during mineralization of cultured human periodontal ligament cells. J Periodontal Implant Sci 41: 30-43.

51. Fujita T, Otsuka-Tanaka Y, Tahara H, Ide T, Abiko Y, et al. (2005) Establishment of immortalized clonal cells derived from periodontal ligament cells by induction of the hTERT gene. J Oral Sci 47: 177-184.

52. Elçin YM, Inanç B, Elçin AE (2014) Differentiation of Human Embryonic Stem Cells on Periodontal Ligament Fibroblasts. Methods Mol Biol.

53. Gronthos S, Zannettino AC, Hay SJ, Shi S, Graves SE, et al. (2003) Molecular and cellular characterisation of highly purified stromal stem cells derived from human bone marrow. J Cell Sci 116: 1827-1835.

54. Ivanovski S, Haase HR, Bartold PM (2001) Expression of bone matrix protein mRNAs by primary and cloned cultures of the regenerative phenotype of human periodontal fibroblasts. J Dent Res 80: 1665-1671.

55. Xu Q, Li B, Yuan L, Dong Z, Zhang H, et al. (2014) Combination of platelet-rich plasma within periodontal ligament stem cell sheets enhances cell differentiation and matrix production. J Tissue Eng Regen Med. 